\title{
Spin polarization of dilute magnetic semiconductors under optical excitation of impurity levels
}

\author{
Gorley ${ }^{1}$ P.M., Mysliuk ${ }^{1}$ O.M., Vieira ${ }^{2}$ M., Horley ${ }^{1,3}$ P.P., \\ Dugaev $^{4}$ V.K. and Barnaś ${ }^{5}$ J.
}

Received: 18.12.2007

${ }^{1}$ Department of Electronics and Energy Engineering, Yuri Fedkovych Chernivtsi
National University, 2 Kotsyubynsky St., 58012 Chernivtsi, Ukraine,
e-mail: semicon@ @ chnu.cv.ua
${ }^{2}$ Dept. de Engenharia Electrotécnica, ISEL, Rua Conselheiro Emídio Navarro,
$1950-062$ Lisbon, Portugal
${ }^{3}$ CFIF, Instituto Superior Técnico, Av. Rovisco Pais, 1049-001 Lisbon, Portugal
${ }^{4}$ Department of Mathematics and Applied Physics, Rzeszów University of
Technology, 6 Powstańców Warszawy St., 35-959 Rzeszów, Poland
${ }^{5}$ Institute of Molecular Physics, Polish Academy of Sciences,
$60-179$ Poznań, Poland

\begin{abstract}
We use the method based on kinetic equations to analyze the influence of intensity and polarization of incident light, concentration of impurities with unfilled $3 \mathrm{~d}$ or $4 \mathrm{f}$ shells and long-term relaxation times of conduction electrons and magnetic ions upon the degree of spin polarization. In case of spatially inhomogeneous stationary states, the distribution of band electrons is shown to oscillate in space, with a transition to spatially homogeneous distribution for infinitely thick sample. It is also demonstrated that the maximum spin polarization degree $\eta_{e}$ for the band electrons can be reached for the thickness of semiconductor film comparable to the diffusion length for the minority carriers.
\end{abstract}

Keywords: optical excitation, spin polarization, spintronics, diluted magnetic semiconductors

PACS: $72.25 . \mathrm{Fe}$, 72.25.Rb, 72.25.-b, 78.20.Bh, 78.40.Fy

UDC: 535.568

\section{Introduction}

One of the most efficient methods for creating spin-polarized electrons in semiconductors and manipulating their spin state is optical absorption of circularly polarized light [1-4]. Recent interest in this method has been mainly due to its possible applications in spintronics [3, 5], for which efficient techniques of generating spin-polarized electrons in semiconductors are of crucial importance. The optical techniques become especially important when the electrical injection of electrons from ferromagnet to semiconductor is ineffective, since their conductivities are incompatible [6]. 
Optical absorption of circularly polarized light generates nonequilibrium distributions of spin and charge densities and can also induce spin and charge currents [2]. Combining this effect with electric and/or magnetic fields applied to semiconductor, one can efficiently manipulate the electron spin and spin currents.

The processes of optical excitation are associated with the carrier relaxation, which includes various relaxation channels. A lot of researches have been carried out on various spin relaxation mechanisms in semiconductors under different conditions (see, e.g., [1, 7, 8] and references therein). In this work we involve the carrier relaxation through partial relaxation times related to different relaxation mechanisms. Among them, we take into account a long-term relaxation (LTR) of electrons localized at impurities. We formulate a problem of nonequilibrium stationary state created by optical excitation in the case of several relaxation channels. We consider a model semiconductor with impurities and assume that the optical excitation brings the electrons from deep impurity levels to the conduction band. It should be noted that the electron transitions of this type could be more effective than those from the valence bands to the conduction one, especially in case of indirect interband transitions like those occurring in $\mathrm{Si}$.

The approach commonly used for describing nonequilibrium properties of semiconductors under conditions of optical generation of carriers is based on the kinetic equations [9]. We use this method in order to describe spin dynamics in the semiconductor with deep impurities. In the absence of spin-polarization processes, the method has previously been used to describe photorefractive effects in InP doped with Fe [10]. It has also been employed in the problem of spin-current generation in the spin-polarized p-n junctions and solar cells $[11,12]$. The case of stationary and spatially homogeneous semiconductor subjected to circularly polarized light has been studied by us in Ref. [13]. In this work we consider spatially inhomogeneous states in a semiconductor whose surface is illuminated by light.

Our considerations apply to a large class of semiconductors with magnetic impurities. Such systems are of current interest due to ferromagnetic ordering of the magnetic moments at low temperatures. The role of optical excitation is then double. First, the excitation of free carriers leads to increasing indirect interaction between the localized moments $[14,15]$ and, second, the optically induced polarization of electron gas favours some orientations of magnetic moments of the impurities. The latter corresponds to the magnetic polaronic effect [16, 17].

As said above, we consider a semiconductor with deep impurity levels [13] and describe the dynamics of non-equilibrium systems of spin-polarized electrons in the conduction band and electrons localized at the impurity levels created by elements with unfilled $3 \mathrm{~d}$ or $4 \mathrm{f}$ shells. We assume that the semiconductor is illuminated by polarized light of the frequency that corresponds to the conditions of absorption by the impurity. The concentrations of spin-up $(\uparrow)$ and spin-down $(\downarrow)$ electrons in the conduction band, as well as those located at the impurity levels, can be described by the equations including onedimensional continuity equation and the Gauss's law. A presence of the LTR is taken into 
account for the band electrons and the magnetic ions, together with the effect of thermal ionization at the impurity level and the spin-spin interaction of the band and impurity electrons. Both the drift and diffusion terms are considered for the total current density. In our previous works $[13,18]$ we have studied in detail the influence of the different parameters on the stationary spatially homogeneous distribution of the conduction electrons and their spin polarization. In this study we present results on the spin polarization degree of the band electrons $\left(\eta_{e}\right)$ and the magnetic ions $\left(\eta_{i}\right)$ for the cases of stationary and spatially inhomogeneous distributions.

The paper is organized as follows. Basic theoretical considerations are outlined in section 2. The main numerical results are presented in section 3. Final conclusions are given in section 4 .

\section{Theory}

We consider the model semiconductor with the shallow impurity levels, which are completely ionized at the operation temperatures. These levels are created by donor and acceptor impurities with the concentrations $\mathrm{N}_{\mathrm{d}}$ and $\mathrm{N}_{\mathrm{a}}$, respectively. An additional impurity with the unfilled $3 d$ or $4 f$ electron shell (the concentration $\mathrm{N}_{\mathrm{T}}$ ) is introduced to create a deep level in the band gap. Such a situation is typical for GaAs:Mn, GaN:Mn and InP:Fe systems $[10,19,20]$. To be more specific, we assume that the conductivity of the semiconductor under consideration is of n-type and its band structure is like that of InP:Fe [10, 20]. In this compound, the iron impurity embedded in the tetragonal indium phosphate lattice can exist in two different states: $\mathrm{Fe}^{2+}$, with the equilibrium concentration of the localized electrons $\mathrm{n}_{\mathrm{T} 0}$, and $\mathrm{Fe}^{3+}$, with the equilibrium concentration of the localized holes, $\mathrm{p}_{\mathrm{T} 0}$.

Let the semiconductor is illuminated by circularly polarized light with the frequency corresponding to the impurity absorption and enabling transitions between the deep level and the conduction band. Then the linear transport of spin-polarized electrons along the $\mathrm{z}$ axis can be described by the following equations [13, 21]:

$$
\begin{aligned}
\frac{\partial N_{\uparrow}}{\partial t}=-\left(S I_{L}+\sigma\right) N_{\uparrow}-\beta_{\uparrow} N_{\uparrow}+\beta_{\downarrow} N_{\downarrow}+\gamma_{\uparrow} n_{\uparrow}\left(N_{T}-N\right), \\
\frac{\partial N_{\downarrow}}{\partial t}=-\left(S I_{R}+\sigma\right) N_{\downarrow}-\beta_{\downarrow} N_{\downarrow}+\beta_{\uparrow} N_{\uparrow}+\gamma_{\downarrow} n_{\downarrow}\left(N_{T}-N\right), \\
\frac{\partial n_{\uparrow}}{\partial t}=\left(S I_{L}+\sigma\right) N_{\uparrow}-\alpha_{\uparrow} n_{\uparrow}+\alpha_{\downarrow} n_{\downarrow}-\gamma_{\uparrow} n_{\uparrow}\left(N_{T}-N\right)+\frac{1}{q} \frac{\partial j_{\uparrow}}{\partial z}, \\
\frac{\partial n_{\downarrow}}{\partial t}=\left(S I_{R}+\sigma\right) N_{\downarrow}-\alpha_{\downarrow} n_{\downarrow}+\alpha_{\uparrow} n_{\uparrow}-\gamma_{\downarrow} n_{\downarrow}\left(N_{T}-N\right)+\frac{1}{q} \frac{\partial j_{\downarrow}}{\partial z}, \\
\quad \frac{\partial E}{\partial z}=\frac{q}{\varepsilon \varepsilon_{0}}\left(N_{D}-N_{A}-N-n\right),
\end{aligned}
$$




$$
j_{\uparrow, \downarrow}=q\left(n_{\uparrow, \downarrow} \mu_{n} E+D_{n} \frac{\partial n_{\uparrow, \downarrow}}{\partial z}\right),
$$

where

$$
\begin{gathered}
n=n_{\uparrow}+n_{\downarrow}, \quad N=N_{\uparrow}+N_{\downarrow}, \quad n_{T 0}=N_{D}-N_{A}, n_{T 0}+p_{T 0}=N_{T}, \quad N+P_{T}=N_{T}, \\
V_{0}=\int_{0}^{l} E(z, t) d z,
\end{gathered}
$$

and $\mathrm{V}_{0}$ denotes the bias voltage. Differentiating Eq. (5) over time and using Eqs. (1)-(4), one obtains

$$
\frac{\partial E}{\partial t}=\frac{1}{\varepsilon \varepsilon_{0}}\left(j_{0}-j_{n}\right)
$$

with the total electric current $j_{n}=j_{\uparrow}+j_{\downarrow}$ and the time-dependent, though coordinateindependent, integration constant $j_{0}$ representing the current through the sample in spatially homogeneous case. It is worth mentioning that the system of Eqs. (1)-(4) is written for the case when the incident light flux is completely absorbed by the sample surface. Using the dimensionless variables

$$
\begin{gathered}
\bar{n}=\frac{n_{\uparrow}+n_{\downarrow}}{N_{T}}, \bar{n}_{-}=\frac{n_{\uparrow}-n_{\downarrow}}{N_{T}}, \bar{N}=\frac{N_{\uparrow}+N_{\downarrow}}{N_{T}}, \\
\bar{N}_{-}=\frac{N_{\uparrow}-N_{\downarrow}}{N_{T}}, \bar{E}=\frac{E}{E_{0}}, \tau=t \sigma, x=\frac{z}{l},
\end{gathered}
$$

and

$$
\begin{aligned}
& k=\frac{1}{2}\left(I_{L}^{*}-I_{R}^{*}\right), \quad k_{R}=\frac{1}{2}\left(I_{L}^{*}+I_{R}^{*}\right)+1, \\
& d_{\alpha}^{ \pm}=\Delta_{\alpha}\left(z_{\alpha} \pm 1\right), \quad d_{\beta}^{ \pm}=\Delta_{\beta}\left(z_{\beta} \pm 1\right), \quad d_{\gamma}^{ \pm}=\frac{1}{2} \Delta_{\gamma}\left(z_{\gamma} \pm 1\right), \\
& z_{\alpha}=\alpha_{\downarrow} / \alpha_{\uparrow}, \quad z_{\beta}=\beta_{\downarrow} / \beta_{\uparrow}, \quad z_{\gamma}=\gamma_{\downarrow} / \gamma_{\uparrow}, \\
& \Delta_{\alpha}=\alpha_{\uparrow} / \sigma, \quad \Delta_{\beta}=\beta_{\uparrow} / \sigma, \quad \Delta_{\gamma}=\gamma_{\uparrow} N_{T} / \sigma, \\
& I_{L, R}^{*}=S I_{L, R} / \sigma, \quad R=\left(N_{D}-N_{A}\right) / N_{T}, \\
& a_{1}=\frac{\mu_{n} q N_{T}}{\sigma \varepsilon \varepsilon_{0}}, \quad a_{2}=\frac{\mu_{n} E_{0}}{l \sigma}, \quad a_{3}=\frac{D_{n}}{l^{2} \sigma},
\end{aligned}
$$

we transform the system of Eqs. (1)-(4) and (9) into

$$
\begin{aligned}
& \frac{\partial N}{\partial \tau}=-k_{R} \bar{N}-k \bar{N}_{-}+(1-\bar{N})\left[d_{\gamma}^{+} \bar{n}-d_{\gamma}^{-} \bar{n}_{-}\right], \\
& \frac{\partial \bar{N}_{-}}{\partial \tau}=-\left[k-d_{\beta}^{-}\right] \bar{N}-\left[k_{R}+d_{\beta}^{+}\right] \bar{N}_{-}-(1-\bar{N})\left[d_{\gamma}^{-} \bar{n}-d_{\gamma}^{+} \bar{n}_{-}\right],
\end{aligned}
$$




$$
\begin{gathered}
\frac{\partial \bar{n}}{\partial \tau}=k_{R} \bar{N}+k \bar{N}_{-}-(1-\bar{N})\left[d_{\gamma}^{+} \bar{n}-d_{\gamma}^{-} \bar{n}_{-}\right]+a_{1} \bar{n}(R-\bar{N}-\bar{n})+a_{2} \bar{E} \frac{\partial \bar{n}}{\partial x}+a_{3} \frac{\partial^{2} \bar{n}}{\partial x} \\
\frac{\partial \bar{n}_{-}}{\partial \tau}=k \bar{N}+k_{R} \bar{N}_{-}+d_{\alpha}^{-} \bar{n}-d_{\alpha}^{+} \bar{n}_{-}+(1-\bar{N})\left[d_{\gamma}^{-} \bar{n}-d_{\gamma}^{+} \bar{n}_{-}\right]+ \\
a_{1} \bar{n}_{-}(R-\bar{N}-\bar{n})+a_{2} \bar{E} \frac{\partial \bar{n}_{-}}{\partial x}+a_{3} \frac{\partial^{2} \bar{n}_{-}}{\partial x} \\
\frac{\partial \bar{E}}{\partial \tau}=a_{1}\left[\bar{n}^{0}-\bar{n} \bar{E}-\frac{a_{3}}{a_{2}} \frac{\partial \bar{n}}{\partial x}\right]
\end{gathered}
$$

Balance equations (12) to (16) allow to determine the dependence of $\bar{n}, \bar{n}_{-}, \bar{N}$, $\bar{N}_{-}$and $E$ on the governing parameters $\left(I_{L, R}^{*}, R, \Delta_{\alpha}, \Delta_{\beta}, \Delta_{\gamma}, z_{\alpha}, z_{\beta}\right.$ and $\left.z_{\gamma}\right)$. The physical quantities entering Eqs. (12)-(16) are as follows: the intensities $I_{L}{ }^{*}$ and $I_{R}{ }^{*}$ of the left- and right-hand circularly polarized light waves, the cross-section $S$ of the ionization centres, the thermal excitation rate $\sigma$, the inverse LTR time for the magnetic impurity subsystem $\beta_{\uparrow, \downarrow}$, the electron recombination rate $\gamma_{\uparrow, \downarrow}$ via the impurity centres, the inverse LTR times for the conduction electrons $\alpha_{\uparrow, \downarrow}$, the electron drift mobility $\mu_{n}$ (assumed to be independent of spin orientation), linear unit of the scale $l$ and, finally, the stationary inner electric field $E_{0}$. The other parameters have their usual meaning.

In case of a stationary, spatially homogeneous solution of Eqs. (12)-(16) we find that the concentration $\bar{n}^{0}$ is a solution of the equation [13]

$$
\left(\bar{n}^{0}\right)^{3}+a_{s}\left(\bar{n}^{0}\right)^{2}+b_{s} \bar{n}^{0}-c_{s}=0,
$$

where the coefficients $a_{s}, b_{s}$ and $c_{s}$ are specified in Appendix A. The expressions for the remaining variables, $\bar{n}_{-}^{0}, \bar{N}^{0}$, and $\bar{N}_{-}^{0}$, can be calculated using the known solution for $\bar{n}^{0}[13]$.

For the case of stationary and spatially inhomogeneous distributions, one can find that the concentration $\bar{n}$ becomes a solution of the equations

$$
\begin{aligned}
& \frac{d^{2} \bar{n}}{d x^{2}}-\frac{1}{n}\left(\frac{d \bar{n}}{d x}\right)^{2}+\frac{a_{2}}{a_{3}} \frac{\bar{n}^{0}}{n} \frac{d \bar{n}}{d x}-L_{1}\left(\bar{n}, \bar{n}_{-}\right)=0, \\
& \frac{d^{2} \bar{n}_{-}}{d x^{2}}-\frac{1}{n} \frac{d n}{d x} \frac{d \bar{n}_{-}}{d x}+\frac{a_{2}}{a_{3}} \frac{\bar{n}^{0}}{n} \frac{d \bar{n}_{-}}{d x}-L_{2}\left(\bar{n}, \bar{n}_{-}\right)=0 .
\end{aligned}
$$

The parameters $\bar{N}$ and $\bar{N}_{-}$may be found using the known solutions for $\bar{n}$ and $\bar{n}_{-}$ as

$$
\begin{aligned}
\bar{N} & =1-\frac{c}{a \bar{n}-b \bar{n}_{-}+c}, \\
\bar{N}_{-} & =\frac{1}{k_{R}+d_{\beta}^{+}}\left[\left(-\left(k_{L}-d_{\beta}^{-}\right)+d_{\gamma}^{-} \bar{n}-d_{\gamma}^{+} \bar{n}_{-}\right) \bar{N}-d_{\gamma}^{-} \bar{n}+d_{\gamma}^{+} \bar{n}_{-}\right],
\end{aligned}
$$


where

$$
a=\frac{k_{L}}{k_{R}+d_{\beta}^{+}} d_{\gamma}^{-}+d_{\gamma}^{+}, \quad b=\frac{k_{L}}{k_{R}+d_{\beta}^{+}} d_{\gamma}^{+}+d_{\gamma}^{-}, \quad c=k_{R}-k_{L} \frac{k_{L}-d_{\beta}^{-}}{k_{R}+d_{\beta}^{+}}
$$

and the relations for $L_{1}\left(\bar{n}, \bar{n}_{-}\right)$and $L_{2}\left(\bar{n}, \bar{n}_{-}\right)$are given in Appendix B.

Based on the solutions for $\bar{n}$ and $\bar{n}_{-}$, one can determine the stationary spatially inhomogeneous polarization degree of the band electrons,

$$
\eta_{e}=\frac{\bar{n}_{-}}{\bar{n}} .
$$

The stationary spatially inhomogeneous polarization of electrons at the impurity centres may be derived in a similar way,

$$
\eta_{i}=\frac{\bar{N}_{-}}{\bar{N}} .
$$

As the system given by Eqs. (18) does not allow exact analytical solution, it has been solved numerically, using the Runge-Kutta method of the fourth order [22].

\section{Results and discussion}

The set of governing parameters considered above may be divided into two subsets. The first one would describe external parameters such as the polarization type and the intensity of the light wave $I_{L, R}^{*}$, as well as the type and concentration of the magnetic impurities (accounted via the parameter $\left.R=\left(N_{D}-N_{A}\right) / N_{T}\right)$. The second subset includes internal parameters of the semiconductor $\left(z_{\alpha}, z_{\beta}, z_{\gamma}, \Delta_{\alpha}, \Delta_{\beta}\right.$ and $\left.\Delta_{\gamma}\right)$. In our calculations we have taken the values $\gamma=4 \times 10^{-14} \mathrm{~m}^{3} / \mathrm{s}, \quad \sigma=8 \times 10^{4} \mathrm{~s}^{-1}, \quad N_{D}-N_{A}=2 \times 10^{22} \mathrm{~m}^{-3}$, $N_{T}=7 \times 10^{22} \mathrm{~m}^{-3}$ and $l=10 \mu \mathrm{m}$ (see $[10,19]$ ). The values of $z_{\alpha, \beta, \gamma}$ are characteristic for III-V semiconductors [20]: $z_{\alpha}=2.5, z_{\beta}=4 / 3$ and $z_{\gamma}=1.25$, whereas the parameter $I_{0}^{*}=3$ has been chosen so as to fit the spin polarization degree of electrons $\eta_{e}$ calculated theoretically to the corresponding experimental data $\left(\eta_{e}=0.42 \pm 0.08\right.$ for GaSb [23] and $\eta_{e}=0.46 \pm 0.06$ for $\left.\mathrm{Ga}_{0.7} \mathrm{Al}_{0.3} \mathrm{As}[24]\right)$.

Fig. 1 presents the concentration distribution $n$ for the band electrons as a function of distance, starting from the sample surface. As one can see, the distribution in the vicinity of the surface manifests damping oscillations, the amplitude of which coincides with the stationary spatially homogeneous distribution on the back side of the sample (i.e., the side opposite to the illuminated one). Eqs. (12)-(16) form a set of non-linear differential equations. Under significant external influence they may involve self-organization processes [26], which appear in the system as oscillatory states observable in Fig. 1. It is worth emphasizing that, for the fixed values of parameters, the linearly polarized light increases the concentration of band electrons by a factor of three, due to transitions between the impurity level and the conduction band. At the same time, the left-handed circularly polarized 
light enables obtaining two-times higher concentration values, while the carrier concentration obtained under the action of the right-handed circularly polarized illumination increases only by $150 \%$.

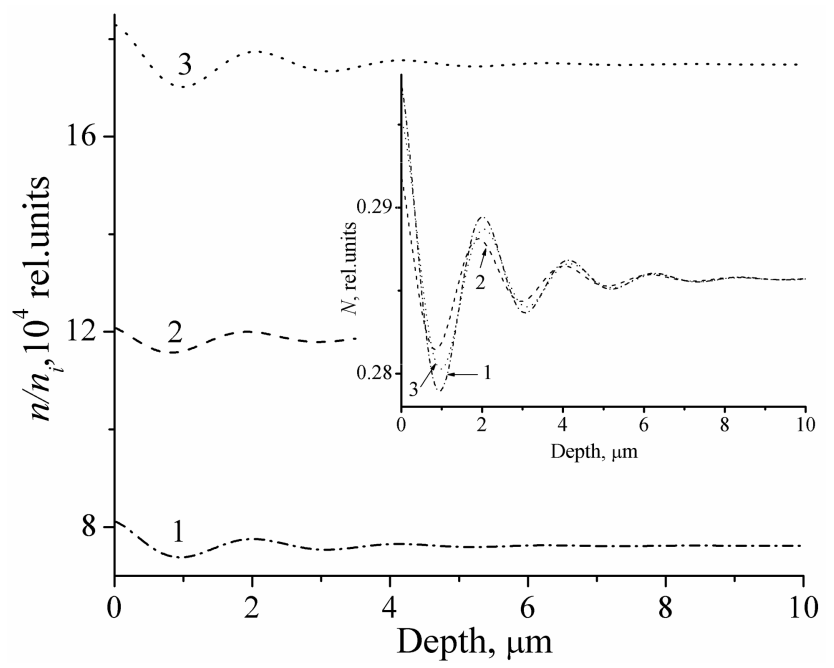

Fig. 1. Spatial distributions of electron concentration in the conduction band for different polarizations of the incident light wave: $I_{R}{ }^{*}, I_{L}{ }^{*}$ and $I_{L R}^{*}=3$ for the curves 1,2 and 3 , respectively. The inset represents spatial distributions of electron concentration at the impurity level for the same polarizations of the incident light. The intrinsic concentration for $\mathrm{InP}$ is $n_{i}=1.3 \times 10^{13} \mathrm{~m}^{-3}[25]$.

It is worth mentioning that the data presented in Fig. 1 can be interpreted as a concentration distribution along the film thickness $(n(x))$, whenever the film growth process allows controlling of the latter thickness. As seen from Fig. 1, the space distributions $\bar{n}(x)$ and $\bar{N}(x)$ for InP:Fe may be roughly divided into three regions, depending on the sample thickness: (1) thin films $(l<10 \mu \mathrm{m}$; distance-damping concentrations), (2) samples of intermediate thicknesses $(15<l<75 \mu \mathrm{m}$; oscillatory concentration distributions), and (3) thick samples ( $l>80 \mu \mathrm{m}$, both $\bar{n}(x)$ and $\bar{N}(x)$ change insignificantly with coordinate and tend to their spatially homogeneous values in the limiting case of infinitely thick samples [13]).

Our analysis has shown that the functions $\bar{n}(x)$ and $\bar{n}_{-}(x)$ could be approximated with a marginal error of $2 \%$ for any incident light polarization by the formula

$$
n(x)=P_{1} e^{-P_{2} x} \cos \left(P_{3} x\right)+P_{4}+P_{5} \sqrt{x} .
$$

For more particular cases, one can also use the following formulae:

(a) right-handed and linear light polarizations

$$
\bar{n}_{-}(x)=B_{1} e^{-B_{2} x} \cos \left(B_{3} x\right)+B_{4}-B_{5} x+B_{6} x^{2},
$$

(b) left-handed light polarization

$$
\bar{n}_{-}(x)=B_{1} e^{-B_{2} x} \sin \left(B_{3} x\right)+B_{4}-B_{5} x+B_{6} x^{2} .
$$

The values of the parameters appearing in Eqs. (23)-(25) are summarized in Table 1. We have verified the validity of our parameters $P_{n}$ and $B_{n}(n=1$ to 6$)$ when substituting them into Eqs. (23)-(25) and (19). The results obtained in this way have been compared to those of numerical calculations, resulting in the maximum mismatch of $1.5 \%$. 
The positions of broad $\eta_{e}$ maxima seen from Fig. 2 correlate with the experimental value of the diffusion length $l_{D}$ [22] and show no dependence on the incident wave polarization. The largest $\eta_{e}$ value for the fixed parameters is achieved for the left-handed circularly polarization of light, the intermediate one - for the linear polarization and the lowest - for the right-handed circular polarization. In the limiting case $l / l_{D} \rightarrow \infty$ the spin polarization degree $\eta_{e}$ tends to its stationary homogeneous value $\eta_{e}{ }^{0}$ [13].

Table 1. The parameters entering Eqs. (23)-(25).

\begin{tabular}{|l|l|l|l|}
\hline & $\mathrm{I}_{\mathrm{R}}=3$ & $\mathrm{I}_{\mathrm{L}}=3$ & $\mathrm{I}_{\mathrm{LR}}=3$ \\
\hline $\mathrm{P}_{1} \times 10^{-7}$ & 0.8842 & 0.4724 & 1.475 \\
\hline $\mathrm{P}_{2}$ & 0.6326 & 0.3686 & 0.540 \\
\hline $\mathrm{P}_{3}$ & 3.0357 & 3.1983 & 2.988 \\
\hline $\mathrm{P}_{4} \times 10^{-5}$ & 1 & 2 & 3 \\
\hline $\mathrm{P}_{5} \times 10^{-8}$ & 1.799 & -5.426 & -0.8725 \\
\hline $\mathrm{B}_{1} \times 10^{-7}$ & 2.368 & -6.210 & 5.131 \\
\hline $\mathrm{B}_{2}$ & 0.3737 & 0.8619 & 0.4110 \\
\hline $\mathrm{B}_{3}$ & 3.070 & 2.5254 & 3.0115 \\
\hline $\mathrm{B}_{4} \times 10^{-5}$ & 0.6386 & 1 & 1 \\
\hline $\mathrm{B}_{5} \times 10^{-8}$ & 0.3938 & 1.079 & 0.9256 \\
\hline $\mathrm{B}_{6} \times 10^{-11}$ & 0.9701 & 3.572 & 2.740 \\
\hline
\end{tabular}

Fig. 3 demonstrates that the increase in the LTR for the spin sub-system of the band electrons occurred under left-handed circularly polarized illumination leads to increasing $\eta_{e}$, while the linearly or right-handed circularly polarized incident waves decrease this value. For the right-handed light the changes in the spin polarization degree are more pronounced than those obtained in case of the linear polarization. The dependence of the spin polarization degree $\eta_{i}$ on the incident-light polarization manifests a similar character and differs only by magnitude.

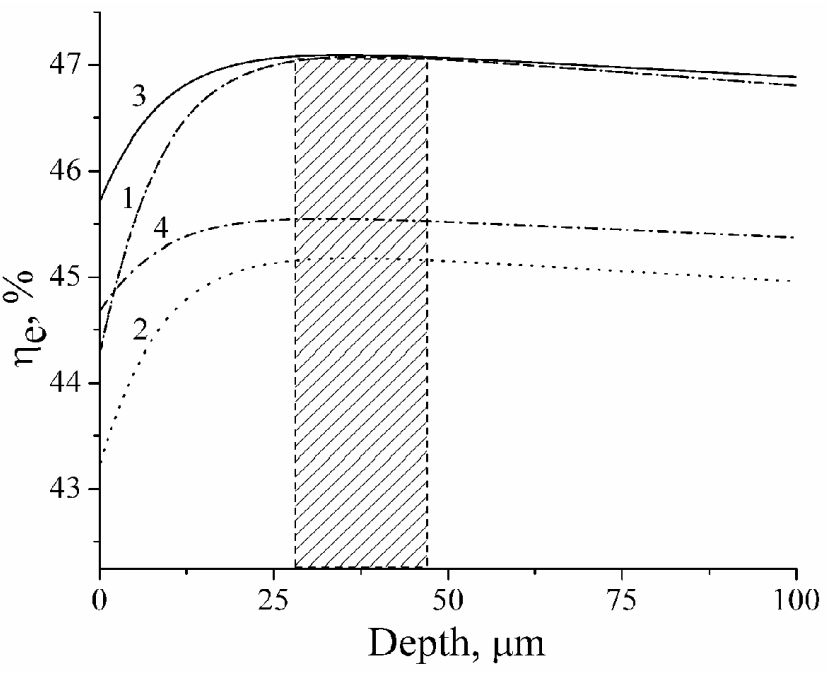

Fig. 2. Spatial distributions of spin polarization degree of the band electrons for different polarizations of the incident light wave: curve 1 corresponds to $I_{0}^{*}=0$ and curves 2,3 and 4 to $I_{R}^{*}, I_{L}^{*}$ and $I_{L R}^{*}=3$, respectively. 

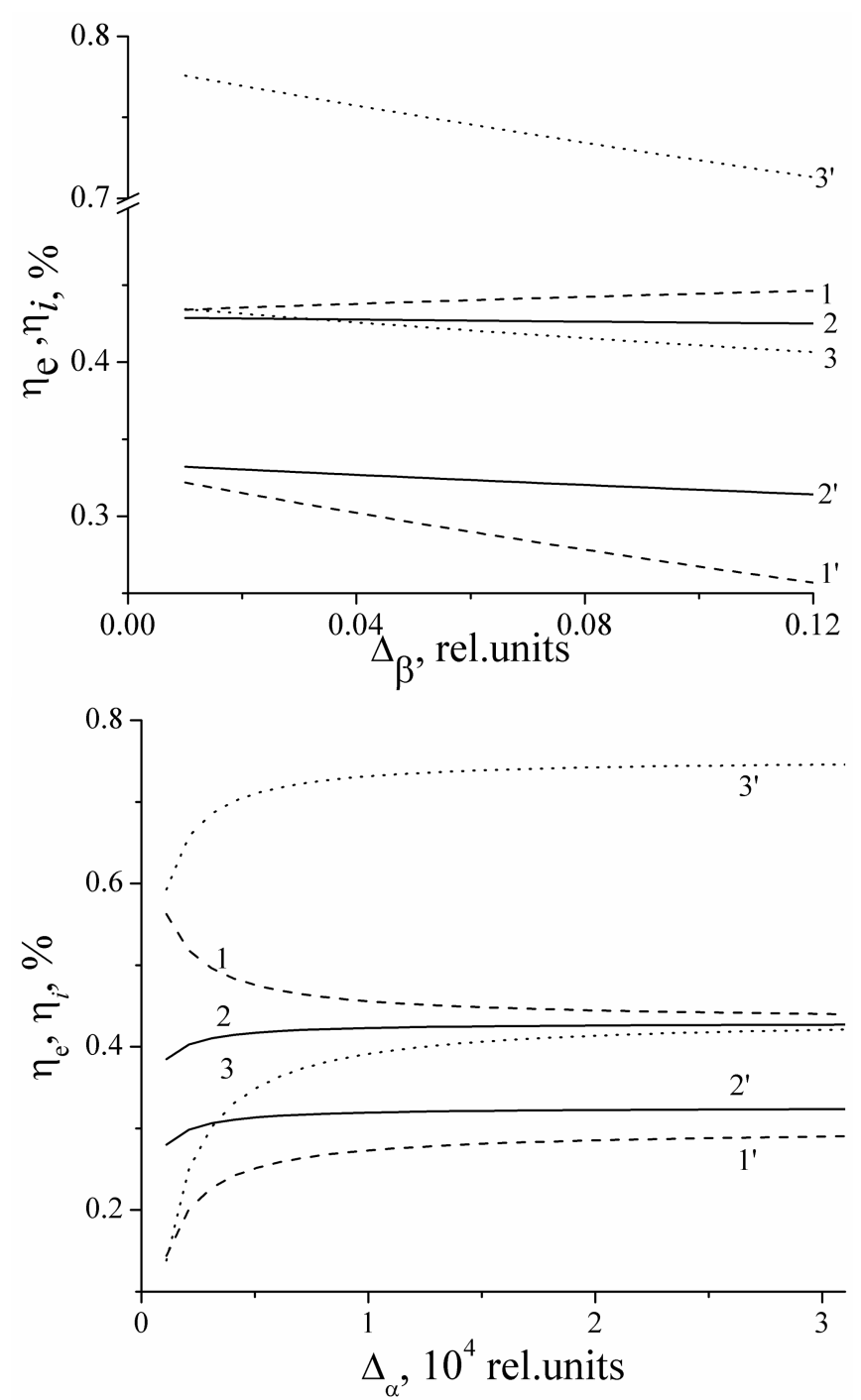

Fig. 3. Spin polarization degrees for the band $\left(\eta_{e}\right)$ and impurity $\left(\eta_{i}\right.$, primed numerals) electrons as functions of LTR of the spin subsystem for different polarizations of the incident light wave: $1,1^{\prime}-I_{L}^{*}$;

2, 2 - $-I_{L R}$;

$3,3^{\prime}-I_{R}{ }^{*} \cdot \eta_{i}$ represents the absolute value for the case of left-handed polarization of the incident light.

Fig. 4. Dependences of spin polarization degree for the band $\left(\eta_{e}\right)$ and impurity ( $\eta_{i}$, primed numerals) electrons on LTR of the spin subsystem of magnetic ions for different polarizations of the incident light wave:

$1,1^{\prime}-I_{L}^{*}$;

2, $2 '-I_{L R}$;

$3,3^{\prime}-I_{R}$.

The absolute values of the spin polarization degree $\eta_{i}$ are plotted for the lefthanded incident polarization.

Finally, it follows from Fig. 4 that the increase in the LTR time for the spin subsystem of magnetic ions makes the values of spin polarization degree $\eta_{e}$ closer to each other. This value decreases with increasing LTR for the left-handed polarization. On the contrary, it increases for the right-handed circularly polarized light. At last, the $\eta_{e}$ value for the linearly polarized light remains practically invariable when the LTR changes.

\section{Conclusions}

We have described stationary spatially inhomogeneous dependence of the concentrations of spin-polarized electrons and the polarization degrees $\eta_{e}$ and $\eta_{i}$ for the conduction band and the magnetic impurities. Our results refer to the diluted magnetic semiconductors subjected to influence of polarized light. We have used the numerical results obtained with our analytical expressions for describing spatially oscillating distributions of the concentrations of band electrons under the conditions of different polarizations and con- 
stant intensity of the incident light. It has been shown that the spin polarization degree $\eta_{e}$ of the conduction-band electrons as a function of sample thickness represents a curve with a peak, the position of which corresponds to the film thickness close to the diffusion length of minority carriers.

\section{Acknowledgments}

This work is partly supported by the STCU Grant No. 3098 of Ukraine and the FCT Grant PTDC/FIS/70843/2006 of Portugal. The authors also acknowledge financial support from the Polish Ministry of Science and Higher Education under the research projects implemented in 2006-2009 and 2007-2010.

\section{Appendix A}

The coefficients in Eq. (17) may be written as

$$
\begin{aligned}
& a_{s}=\frac{1}{z_{\gamma} \Delta_{\gamma}^{2}}\left[d_{\gamma}^{+}\left(b_{11}+c_{11}\right)+d_{\gamma}^{-}\left(a_{22}-b_{22}\right)\right], \\
& b_{s}=\frac{1}{z_{\gamma} \Delta_{\gamma}^{2}}\left[d_{\gamma}^{+} a_{11}-d_{\gamma}^{-} c_{22}+b_{11} c_{11}+a_{22} b_{22}\right], \quad c_{s}=\frac{1}{z_{\gamma} \Delta_{\gamma}^{2}}\left[a_{11} c_{11}+a_{22} c_{22}\right],
\end{aligned}
$$

where

$$
\begin{aligned}
& a_{11}=-\left[k_{R}+k \frac{z_{\beta}-1}{z_{\beta}+1}\right] R, \quad a_{22}=k \frac{d_{\alpha}^{-}}{d_{\beta}^{+}}-d_{\gamma}^{-}(1-R), \\
& b_{11}=k_{R}-k\left(\frac{d_{\alpha}^{-}}{d_{\beta}^{+}}-\frac{z_{\beta}-1}{z_{\beta}+1}\right)+d_{\gamma}^{+}(1-R), \\
& b_{22}=d_{\alpha}^{-}\left[\frac{k_{R}}{d_{\beta}^{+}}+1\right]-k-k_{R} \frac{z_{\beta}-1}{z_{\beta}+1}+d_{\gamma}^{-}(1-R), \\
& c_{11}=d_{\alpha}^{+}\left[\frac{k_{R}}{d_{\beta}^{+}}+1\right]+d_{\gamma}^{+}(1-R), \quad c_{22}=\left[k+k_{R} \frac{z_{\beta}-1}{z_{\beta}+1}\right] R .
\end{aligned}
$$

All the other quantities entering the above formulae are explained in the main text.

\section{Appendix B}

The coefficients in Eq. (18) may be derived as follows:

$$
\begin{aligned}
& L_{1}\left(\bar{n}, n_{-}\right)=\frac{a_{1}}{a_{3}} \bar{n}\left[\frac{c}{a \bar{n}-b \bar{n}_{-}+c}-(1-R+\bar{n})\right], \\
& L_{2}\left(\bar{n}, \bar{n}_{-}\right)=\frac{1}{a_{3}}\left[k_{L}-k_{R} \frac{k_{L}-d_{\beta}^{-}}{k_{R}+d_{\beta}^{+}}+d_{\alpha}^{-} \bar{n}-d_{\alpha}^{+} \bar{n}_{-}-a_{1} \bar{n}_{-}(1-R+\bar{n})\right] \\
& -\frac{c}{a_{3}\left(a \bar{n}-b \bar{n}_{-}+c\right)}\left[k_{L}-k_{R} \frac{k_{L}-d_{\beta}^{-}}{k_{R}+d_{\beta}^{+}}+\left(\frac{k_{R}}{k_{R}+d_{\beta}^{+}}-1\right)\left(d_{\gamma}^{-} \bar{n}-d_{\gamma}^{+} \bar{n}_{-}\right)-a_{1} \bar{n}_{-} .\right.
\end{aligned}
$$




\section{References}

1. Meier F. and Zakharchenya B. P. Optical Orientation. Amsterdam: North-Holland (1984).

2. Ganichev S and Prettl W, 2003. Spin photocurrents in quantum wells. J. Phys.: Condens. Matter. 15: R935 - R983.

3. Zutić I, Fabian S and Das Sarma S, 2004. Spintronics: fundamentals and applications. Rev. Mod. Phys. 76: 323-410.

4. Awschalom D.D. and Samarth N. Semiconductor Spintronics and Quantum Computation. Belin: Springer (2002).

5. Wolf S A, Awschalom D D, Buhrman R A, Daughton J M, von Molnár S, Roukes M L, Chtchelkanova A Y and Treger D M, 2001. Spintronics: A spin-based electronics vision for the future. Science 294: 1488-1495.

6. Schmidt G, Ferrand D, Molenkamp L W, Filip AT and van Wees B J, 2000. Fundamental obstacle for electrical spin injection from a ferromagnetic metal into a diffusive semiconductor. Phys. Rev. B 62: R4790-R4793.

7. Fabian J and Das Sarma S J, 1999. Spin relaxation of conduction electrons. J.Vac. Sci. Technol. B 17: 1708-1715.

8. Vadim I Puller, Lev G Mourokh, Norman J Horing, and Anatoly Yu Smirnov, 2003. Electron spin relaxation in a semiconductor quantum well. Phys. Rev. B 67: 155309155318.

9. Lifshitz E. M. and Pitaevskii L. P. Physical Kinetics. Oxford: Pergamon (1997).

10. Picoli G, Gravey P, Ozcul C and Vieux V, 1989. Theory of two-wave mixing gain enhancement in photorefractive InP:Fe: A new mechanism of resonance. J. Appl. Phys. 66: 3798-3813.

11. Zutić I, Fabian J and Das Sarma S, 2001. Spin injection through the depletion layer: A theory of spin-polarized $p-n$ junctions and solar cells. Phys. Rev. B 64: 121201121205.

12. Zutić I, Fabian J and Das Sarma S, 2001. Proposal for a spin polarized solar battery. Appl. Phys. Lett. 79: 1558-1560.

13. Gorley PM, Dugaev VK, Barnaś J, Horley PP, Mysliuk OM, 2007. Spin polarization and relaxation in a semiconductor with impurity absorption of circularly polarized light. J. Phys.: Condens. Matter. 19: 266205-266214.

14. Ohno H, Chiba D, Matsukura F, Omiya T, Abe E, Dietl T, Ohno Y and Ohtani K, 2000. Electric-Field control of ferromagnetism. Nature 408: 944-946.

15. Boukari H, Kossacki P, Bertolini M, Ferrand D, Cibert J, Tatarenko S, Wasiela A, Gaj J A and Dietl T, 2002. Light and Electric Field Control of Ferromagnetism in Magnetic Quantum Structures. Phys. Rev. Lett. 88: 207204-207208.

16. Torrance JB, Shafer MW and McGuire T R, 1972. Bound Magnetic Polarons and the Insulator-Metal Transition in EuO. Phys. Rev. Lett. 29: 1168-1171.

17. Dietl T and Spałek J, 1983. Effect of thermodynamic fluctuations of magnetization 
on the bound magnetic polaron in dilute magnetic semiconductors. Phys. Rev. B 28: 1548-1563.

18. Gorley P. M., Dugaev V. K., Barnaś J., Vieira M., Horley P. P. and Mysliuk O. M. Novel Semiconductor Materials for Room-Temperature Ferromagnetism. Warrendale: Materials Research Society (2007).

19. Ippolitova G K, Omel'yanovski E M, Pavlov N M, Nashel'skiy A Ya and Yakobson S V, 1977. Behaviour of Fe impurity in InP and influence of covalence on the spectrum of EPR of ion Fe3+ in Td-symmetry compounds. Fiz. Tekhn. Poluprovod. 11: 1315-1320.

20. Pressel K, Bohnert G, Dörnen A, Kaufmann B, Denzel J, and Thonke K, 1993. Optical study of spin-flip transitions at $\mathrm{Fe}^{3+}$ in InP. Phys. Rev. B 47: 9411-9417.

21. Gorley PM, Horley PP, Gonzalez-Hernandez J Vorobiev YuV, 2002. Selforganization processes in semiconductor under photo-induced Gunn effect. Mater. Sci. Eng. B 88: 286-291.

22. Samarskii A. A. and Gulin A. V. Numerical methods. Moscow: Nauka (1999).

23. Parsons RR, 1969. Band-To-Band Optical Pumping in Solids and Polarized Photoluminescence. Phys. Rev. Lett. 23: 1152-1154.

24. Ekimov A I and Safarov V I, 1970. Optical Orientation of Carriers in Interbank Transitions in Semiconductors. Pisma Zhurn. Eksp. Teor. Fiz. 12: 198-201.

25. Pearsall T. P. Properties, Processing and Applications of Indium Phosphide. London: INSPEC, Institution of Electrical Engineers (2000).

26. Haken H. Synergetics. Berlin, Heidelberg, New York: Springer Verlag (1978). 